\title{
Autoeficácia da amamentação pela breastfeeding self-efficacy scale no Brasil: revisão integrativa da literatura
}

\author{
Auto-effectiveness scale of self-effectiveness breeding in Brazil: integration of the literature \\ review
}
Autoeficacia de la amamentación por breastfeeding self-efficacy scale en Brasil: revisión integral de la literatura

Marcos Rafael Campos Lopes ${ }^{1}$; Rodolfo Martins Magalhães Neto ${ }^{1}$; Rosineide de Souza Broges ${ }^{1}$; Sâmylla Kelly Carvalho Dutra ${ }^{1}$; Orácio Carvalho Ribeiro Júnior ${ }^{1}$.

\section{RESUMO}

Objetivo: Analisar a produção científica brasileira sobre a avaliação da autoeficácia da amamentação pela Breastfeeding self-efficacy scale. Métodos: Trata-se de uma Revisão Integrativa da Literatura com busca por artigos científicos disponíveis nas bases de dados Scientific Electronic Library Online (SCIELO) e Literatura Latino-Americana em Ciências da Saúde (LILACS) e Literatura Internacional em Ciências da Saúde (MEDLINE). Resultados: Através de 6 artigos analisados foi possível identificar que a aplicação da Breastfeeding self-efficacy scale é válida em diferentes contextos no que tange à apreensão da autoeficácia do aleitamento materno. Conclusão: A partir da realização da presente pesquisa, foi possível perceber que a versão brasileira da BSES é um instrumento de relevância e perfeitamente aplicável no que diz respeito à apreensão da autoeficácia de mães para amamentar, e que pode servir como importante instrumento tanto para a prática assistencial, quanto para a pesquisa.

Palavras-chave: Aleitamento Materno, Autoeficácia, Amamentação, Confiança.

\begin{abstract}
Objective: To analyze Brazilian scientific production on the evaluation of breastfeeding self-efficacy by Breastfeeding self-efficacy scale. Methodology: This is an integrative review of the literature with a search for scientific articles available in the Scientific Electronic Library Online (SCIELO) and Latin American Literature in Health Sciences (LILACS) and International Literature in Health Sciences (MEDLINE), from January 2009 to June 2018. Results: Through 6 analyzed articles it was possible to identify that the application of Breastfeeding self-efficacy scale is valid in different contexts regarding the apprehension of the self-efficacy of breastfeeding. Conclusion: Based on the present research, it was possible to perceive that the Brazilian version of BSES is an instrument of relevance and perfectly applicable with regard to the apprehension of the self-efficacy of mothers to increase, and that can serve as an important instrument both for practice, how much, for research.
\end{abstract}

Key words: Breastfeeding, Self-efficacy, Confidence.

${ }^{1}$ Centro Universitário do Norte (UNINORTE), Manaus-Amazonas. *Email: o_ra_cio13@hotmail.com 


\section{RESUMEN}

Objetivo: Analizar la producción científica brasileña sobre la evaluación de la autoeficacia de la lactancia materna por el amamantamiento de la Breastfeeding self-efficacy scale. Metodología: Se trata de una revisión integrativa de la literatura con búsqueda de artículos científicos disponibles en las bases de datos Scientific Electronic Library Online (SCIELO) y Literatura Latinoamericana en Ciencias de la Salud (LILACS) y Literatura Internacional en Ciencias de la Salud (MEDLINE), en el período enero de 2009 a junio de 2018. Resultados: A través de 6 artículos analizados fue posible identificar que la aplicación del Breastfeeding self-efficacy scale es válida en diferentes contextos en lo que se refiere a la aprehensión de la autoeficacia de la lactancia materna. Conclusión: A partir de la realización de la presente investigación, fue posible percibir que la versión brasileña de la BSES es un instrumento de relevancia y perfectamente aplicable en lo que se refiere a la aprehensión de la autoeficacia de madres para amentar, y que puede servir como instrumento importante tanto para la práctica asistencial, como para la investigación.

Descriptores: Lactancia Materna, Autoeficacia, Lactancia, Confianza.

\section{INTRODUÇÃO}

O conceito de amamentação vai muito além do simples ato de alimentar o bebê, é um método que além de promover a saúde física, mental e psíquica do lactente e da nutriz, também é o que mais previne mortes infantis. É essencial e de suma importância que seu início seja nas primeiras horas de vida, e mantido sob livre demanda, tendo o cuidado de evitar o uso de bicos e chupetas. Segundo Brasil (2015) alimentar é nutrir a criança, com repercussão no estado nutricional, em sua habilidade de construir barreiras imunológicas, em sua fisiologia e em sua saúde a longo prazo. A Organização Mundial da Saúde (OMS) recomenda amamentação exclusiva durante os primeiros seis meses de vida e, a partir dessa idade é possível a adição de comida sólida, como por exemplo, frutas e legumes triturados, devendo ser introduzidos como complemento da amamentação até aos dois anos de vida ou mais.

O leite materno é o alimento ideal, ele fornece todos os nutrientes para um desenvolvimento saudável, é seguro e contém anticorpos que ajudam a proteger a criança das doenças infantis comuns. Está prontamente disponível e acessível, o que ajuda a garantir que a criança tenha uma nutrição adequada, além disso, a amamentação exclusiva está associada há um método natural de controle da natalidade. Outros aspectos relacionados aos benefícios da amamentação estão o de prevenir determinados tipos de câncer na mulher, infecções na infância e na vida adulta, além de ter repercussão positiva sobre a inteligência do sujeito na vida adulta (NUNES, 2015; VICTORA et al., 2016).

Um dos problemas que estão diretamente relacionados à amamentação é a ausência desta e/ou o desmane precoce que constitui problema de saúde pública no Brasil e no mundo com consequências para o binômio mãe-filho, além de efeitos negativos para a sociedade e os serviços de saúde, sendo associados como características sociodemográficas maternas, características do lactente e escolaridade do companheiro, história obstétrica e à amamentação (MORAES et al., 2016).

Dados da última pesquisa sobre prevalência de aleitamento materno exclusivo no Brasil mostram que o percentual de mães que conseguem manter este tipo de aleitamento é de apenas $36,6 \%$ e, quando considera-se o período até o primeiro ano de vida o percentual fica em $45,4 \%$, mostrando as iniquidades envolvidas nesta temática tão discutida no país, porém, com sérias deficiências operacionais (BOCCOLINI et al., 2017).

A ausência do aleitamento e/ou o desmame precoce repercute não apenas na saúde da criança, mas também interrompe, de forma abrupta, o vínculo mãe e filho, retarda o retorno da mulher às suas condições físicas anteriores à gravidez e afeta o orçamento familiar devido ao custo das formulas lácteas. Um dos 
aspectos que influencia o desmame precoce é a auto eficácia ou confiança materna em sua habilidade para amamentar (ORIÁ; XIMENES, 2009).

Segundo Oriá e Ximenes (2009) a auto eficácia é a mensuração necessária do comportamento humano, dado que as pessoas precisam acreditarem que podem tornar-se praticantes de hábitos saudáveis e assim buscar meios para alcança-los. Com isso a mulher que acredita na sua capacidade de amamentar, denominada de auto eficácia, devera pratica-la antes que a amamentação seja realizada.

Pesquisas apontam que $27 \%$ das mulheres com baixos níveis de confiança na amamentação durante o pré-natal interromperam o aleitamento materno dentro da primeira semana pós-parto. Mulheres com baixo nível de confiança no aleitamento materno possuem 3,1 vezes mais risco de interromper a amamentação do que aquelas que tinham total confiança, mostrando as repercussões que este fator exerce sobre $o$ aleitamento materno e a prática de amamentar (ORIÁ; XIMENES, 2009).

Reconhecendo que o comportamento da mulher diante do aleitamento materno ainda não havia sido estudado dentro da perspectiva da auto eficácia, foi criada no Canadá, uma escala (Breastfeeding SelfEfficacy Scale- BSES) que pudesse avaliar a confiança materna na amamentação. É uma escala tipo Likert, com conteúdo elaborado a partir dos problemas relacionados à prática e duração da amamentação, estratificado em dois domínios: técnico e o pensamento intrapessoal, cada questão apresenta cinco possibilidades de resposta, com escores variando de 1 a 5 . Os escores totais da escala variam de 33 a 165 pontos. Quanto maior o escore, maior a confiança da mulher no seu potencial de amamentar, constituindo, assim, na maior probabilidade de iniciar e manter o aleitamento exclusivo por um período mais longo. Esta escala foi traduzida e validada para vários países do mundo, entre eles o Brasil e desde então tem sido utilizada em vários cenários para avaliar a auto eficácia da amamentação de mulheres brasileiras (ORIÁ; XIMENES, 2009).

$\mathrm{Na}$ atualidade a mulher tem cada vez mais ocupado outros espaços na sociedade que vão muito além do papel materno como por exemplo as atividades laborais, além da maternidade vivenciada de forma precoce que pode estar acompanhada a imaturidade para o desempenho do papel de mãe entre estes 0 ato de amamentar, fatos que podem levar essas mulheres a se sentirem inseguras no processo de amamentação e consequentemente levando ao desmame precoce ou a ausência da amamentação. Nesse sentido existe a necessidade de mensuração da autoeficácia entre puérperas adolescentes e adultas no sentido de melhorar o entendimento dos fatores que levam ao desmame precoce quanto ao sentimento dessas mulheres relacionados ao ato de amamentar. Assim diante da compilação e do desenvolvimento da Breastfeeding selfefficacy scale (BSES) e sua validação no contexto brasileiro, surge uma singular oportunidade de se verificar qual o grau de eficácia de amamentação que é mensurada pela escala BSES, para que se possa traçar um perfil brasileiro de auto eficácia da amamentação, a partir dos achados trazidos pela aplicação dessa escala no diferentes cenários.

Neste contexto, formula-se a seguinte questão: qual a produção cientifica brasileira sobre a avaliação da autoeficácia da amamentação pela Breastfeeding self-efficacy scale?

Assim, este estudo tem por objetivo analisar a produção científica brasileira sobre a avaliação da autoeficácia da amamentação pela Breastfeeding self-efficacy scale.

\section{MÉTODOS}

Trata-se de um estudo do tipo revisão de literatura. Este tipo de estudo busca sintetizar resultados de pesquisas sobre determinado assunto/problema de forma sistematizada e ampla com vistas a conceber um corpo de conhecimentos com diferentes finalidades, desde a análise do plano conceitual até a definição de estratégias para melhorar a prática clínica dos profissionais da saúde (ERCOLE et al., 2014). 
O estudo orientou-se em seis etapas inter-relacionadas e distintas a saber: identificação do tema e seleção da hipótese ou questão de pesquisa; estabelecimento de critérios para inclusão e exclusão de estudos/amostragem ou busca na literatura; definição das informações a serem extraídas dos estudos selecionados/ categorização dos estudos; avaliação dos estudos incluídos; interpretação dos resultados; e apresentação da revisão/síntese do conhecimento (ERCOLE et al., 2014).

A escolha do tema deu-se pela necessidade de mensuração da autoeficácia em puérperas, haja visto a necessidade para um melhor entendimento tanto dos fatores que levam ao desmame precoce, quanto ao sentimento das mulheres relacionado ao ato de amamentar. Assim, definiu-se a seguinte questão norteadora: qual a produção cientifica brasileira sobre a avaliação da autoeficácia da amamentação pela Breastfeeding self-efficacy scale?

Sequencialmente definiu-se como critérios de inclusão: artigos publicados no Brasil no período de janeiro de 2009 a junho de 2018, em língua portuguesa, disponíveis na íntegra, artigos que respondessem a questão norteadora e o objetivo da revisão. Já os critérios de exclusão foram estudos do tipo revisão de literatura, relatos de experiência, estudos de caso, estudos presentes na literatura cinza e artigos repetidos em mais de uma base de dados, neste caso foi considerada somente a primeira vez que apareceu para a análise.

A pesquisa foi realizada por meio do levantamento de artigos indexados nas bases de dados Literatura Latino-Americana em Ciências da Saúde (LILACS), Medical Literature Analysis and Retrieval System Online (MEDLINE) e na Biblioteca Virtual Scientific Electronic Library Online (SciELO) no período de agosto a outubro de 2018.

No que diz respeito às estratégias de busca, foram selecionados os descritores de acordo com sua indexação nos Descritores em Ciências da Saúde (DeCS), entre eles, Aleitamento Materno, Auto eficácia, Amamentação e Confiança. Posteriormente foi realizado o pareamento desses descritores com o operador boleano AND, afim de apreender os artigos que contivessem sempre um e o outro assunto.

Após o processo de busca nas bases de dados foi feita uma seleção inicial dos estudos pela leitura dos títulos e resumos, aplicando os critérios de inclusão e exclusão. Posteriormente procedeu-se à leitura exaustiva dos artigos pré-selecionados para verificar quais iriam compor a amostra final a ser analisada.

Para a coleta dos dados a serem analisados, elaborou-se um quadro sinótipo que comtemplasse as seguintes informações: ano de publicação, base indexadora, revista, título do artigo, autores, objetivo do estudo, metodologia utilizada, principais agravos analisados.

A análise dos estudos selecionados ocorreu de forma descritiva por meio da síntese das informações extraídas, buscando convergências e divergências entre os dados trazidos pelos estudos da amostra final, com o intuito de reunir e consolidar o conhecimento a respeito do problema de pesquisa em questão e verificar a capacidade do BSES para avaliar a autoeficácia da amamentação entre as mulheres brasileiras.

\section{RESULTADOS}

Durante o processo de pesquisa foram identificados 517 artigos nas bases de dados, usando os descritores: Aleitamento materno and autoeficácia, amamentação and confiança. Na base Literatura LatinoAmericana em Ciências da Saúde (LILACS) após a realização do processo de busca foram encontrados 26 artigos, após os critérios de elegibilidade nenhum artigo foi selecionado. Na base MEDLINE - Literatura Internacional em Ciências da Saúde após o processo de busca encontrou-se 382 artigos e após a aplicação dos critérios de elegibilidade nenhum artigo foi selecionado e na base Scientific Electronic Library Online (SCIELO) após a realização do cruzamento dos descritores foram encontrados 109 artigos, sendo que após os critérios de elegibilidade foram selecionados 6 artigos. A amostra final da revisão foi composta por 6 artigos conforme mostra a Figura 1. 
Quando se faz uma análise dos artigos segundo as bases de dados indexadoras, percebe-se que todos os artigos estão publicados em revistas indexadas na base SCIELO, mostrando por um lado a excelência dessa base nas publicações na área de saúde materno infantil, porém, mostra a fragilidade no que diz respeito às bases MEDLINE e LILACS onde nenhuma publicação foi encontrada. Por outro lado, quando se analisa os estudos segundo a revista de publicação, percebe-se que $100 \%$ das revistas onde os artigos estão publicados são da área de enfermagem mostrando o interesse da profissão e da ciência pela temática da amamentação.

Já na análise segundo abordagem metodológica, os dados mostram que todos os estudos selecionados são do tipo observacional e com consequente abordagem quantitativa. No que tange aos anos de publicação, os artigos foram publicados de 2009 a 2017, mostrando que os estudos são recentes e de grande importância para a saúde, conforme evidenciado no Quadro 1.

Figura 1- Fluxograma de seleção dos artigos para a composição da revisão integrativa.

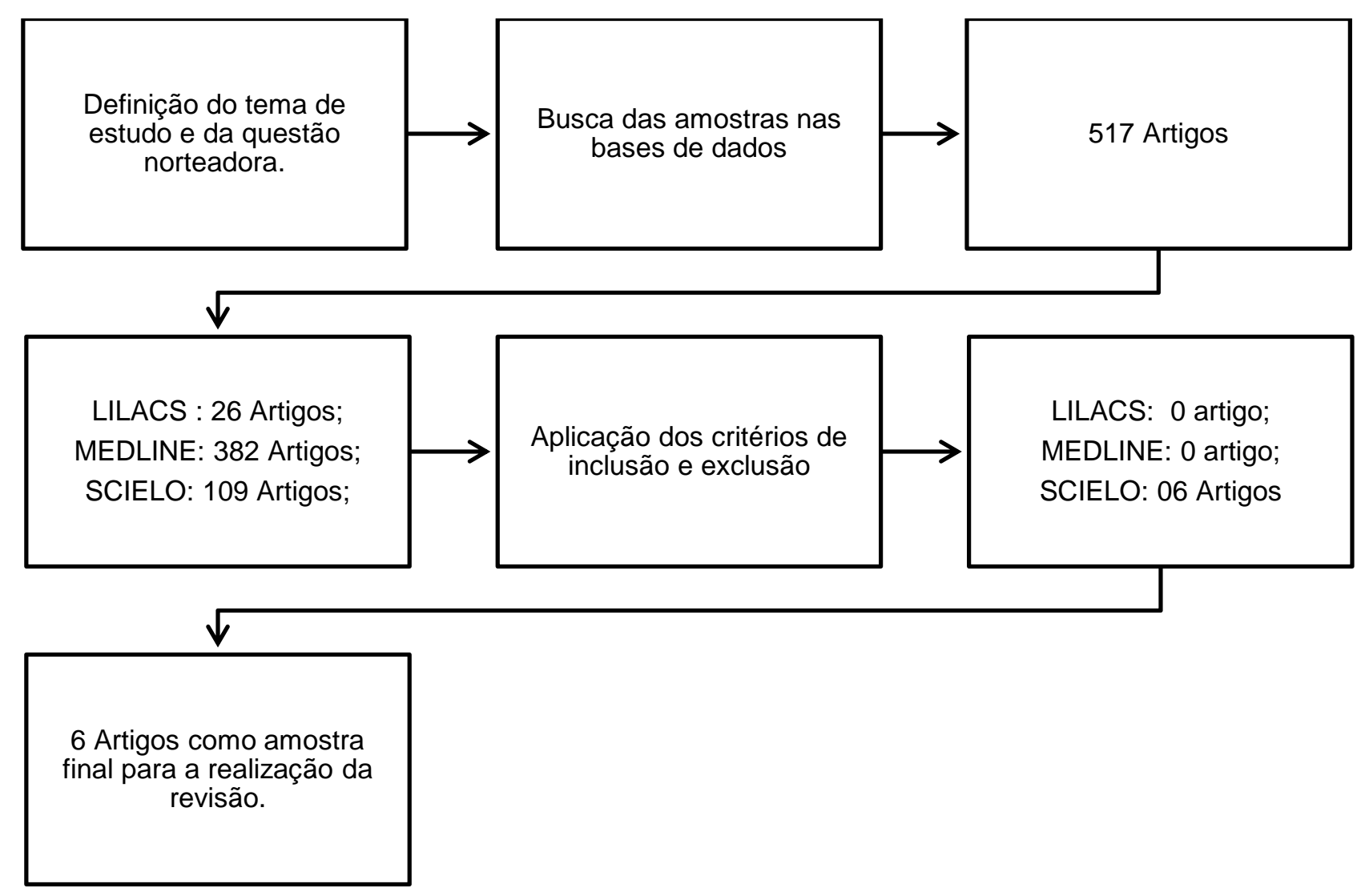

Fonte: autoria própria 
Quadro 1: Caracterização dos estudos a partir dos seguintes tópicos: Base, Revista, Título, Autor (es), Objetivo, Metodologia e Ano.

\begin{tabular}{|c|c|c|c|c|c|c|c|c|}
\hline \multirow{2}{*}{ № } & \multirow{2}{*}{ Autor (es) } & \multirow{2}{*}{ Ano } & \multirow{2}{*}{ Base } & \multirow{2}{*}{ Revista } & \multirow{2}{*}{ Titulo } & \multirow{2}{*}{ Objetivo } & \multicolumn{2}{|c|}{ Metodologia } \\
\hline & & & & & & & Tipo de estudo & Abordagem \\
\hline 1 & $\begin{array}{l}\text { Oriá MOB, } \\
\text { et al. }\end{array}$ & 2009 & SCIELO & $\begin{array}{l}\text { Acta Paulista de } \\
\text { Enfermagem }\end{array}$ & $\begin{array}{l}\text { Tradução e adaptação cultural da } \\
\text { Breastfeeding Self-Efficacy Scale para } \\
\text { o português. }\end{array}$ & $\begin{array}{l}\text { Traduzir e adaptar a Breastfeeding Self- } \\
\text { Efficacy Scale (BSES), para a realidade } \\
\text { cultural do Brasil e avaliar a validade de } \\
\text { conteúdo da versão em português, para que } \\
\text { possa ser utilizada na população brasileira. }\end{array}$ & Transversal & Quantitativa \\
\hline 2 & $\begin{array}{l}\text { Abuchaim } \\
\text { ESV, et al. }\end{array}$ & 2016 & SCIELO & $\begin{array}{l}\text { Acta Paulista } \\
\text { Enfermagem }\end{array}$ & $\begin{array}{l}\text { Depressão pós-parto e autoeficácia } \\
\text { materna para amamentar: prevalência } \\
\text { e associação. }\end{array}$ & $\begin{array}{l}\text { Identificar a prevalência de sintomas de } \\
\text { depressão pós-parto e o nível de } \\
\text { autoeficácia para amamentar, entre } \\
\text { puérperas atendidas num centro de } \\
\text { incentivo ao aleitamento materno, e analisar } \\
\text { possíveis associações. }\end{array}$ & Transversal & Quantitativa \\
\hline 3 & $\begin{array}{l}\text { Mariano } \\
\text { LMB, et al. }\end{array}$ & 2016 & SCIELO & $\begin{array}{l}\text { Texto Contexto } \\
\text { Enfermagem }\end{array}$ & $\begin{array}{l}\text { Aleitamento materno exclusivo e } \\
\text { autoeficácia materna entre mulheres } \\
\text { em situação de violência por parceiro } \\
\text { íntimo. }\end{array}$ & $\begin{array}{l}\text { Busca analisar a prática da amamentação } \\
\text { entre mulheres em situação de violência por } \\
\text { parceiro intimo durante a gestação atual } \\
\text { quanto à duração do aleitamento materno } \\
\text { exclusivo, o nível de autoeficácia para } \\
\text { amamentar e fatores relacionados ao início, } \\
\text { estabelecimento da amamentação e } \\
\text { desame precoce. }\end{array}$ & Transversal & Quantitativa \\
\hline 4 & $\begin{array}{l}\text { Conde RG, } \\
\text { et al. }\end{array}$ & 2017 & SCIELO & $\begin{array}{l}\text { Acta Paulista } \\
\text { Enfermagem }\end{array}$ & $\begin{array}{l}\text { Autoeficácia na amamentação e } \\
\text { duração do aleitamento materno } \\
\text { exclusivo entre mães adolescentes. }\end{array}$ & $\begin{array}{l}\text { Verificar a associação entre a autoeficácia } \\
\text { na amamentação de mães adolescentes e } \\
\text { a duração do aleitamento materno exclusivo } \\
\text { a fim. }\end{array}$ & $\begin{array}{l}\text { Longitudinal } \\
\text { Prospectivo } \\
\text { Observacional } \\
\text { Analítico }\end{array}$ & Quantitativa \\
\hline 5 & $\begin{array}{l}\text { Guimarães } \\
\text { CMS, et al. }\end{array}$ & 2017 & SCIELO & $\begin{array}{l}\text { Texto Contexto } \\
\text { Enfermagem }\end{array}$ & $\begin{array}{l}\text { Comparação da autoeficácia na } \\
\text { amamentação entre puérperas } \\
\text { adolescentes e adultas em uma } \\
\text { maternidade de Ribeirão Preto, Brasil. }\end{array}$ & $\begin{array}{l}\text { Verificar e comparar a autoeficácia na } \\
\text { amamentação } \quad \text { entre } \\
\text { adolescentes e adultas no puérperas } \\
\text { imediato. }\end{array}$ & $\begin{array}{l}\text { Observacional } \\
\text { Transversal } \\
\text { Comparativo }\end{array}$ & Quantitativa \\
\hline 6 & $\begin{array}{l}\text { Conde RG, } \\
\text { et al. }\end{array}$ & 2017 & SCIELO & $\begin{array}{l}\text { Acta Paulista } \\
\text { Enfermagem }\end{array}$ & $\begin{array}{l}\text { Fatores relacionados à autoeficácia na } \\
\text { amamentação no pós-parto imediato } \\
\text { entre puérperas adolescentes. }\end{array}$ & $\begin{array}{l}\text { Verificar a associação entre a autoeficácia } \\
\text { na amamentação e os fatores } \\
\text { sociodemográficos e obstétricos das } \\
\text { adolescentes. }\end{array}$ & $\begin{array}{l}\text { Observacional } \\
\text { Transversal } \\
\text { Descritivo }\end{array}$ & Quantitativa \\
\hline
\end{tabular}

Fonte: Autoria Própria

REAS/EJCH | Vol.Sup.17 | e336 | DOI: https://doi.org/10.25248/reas.e336.2019 Página 6 de 8 


\section{DISCUSSÃO}

No estudo realizado por Oriá e Ximenes (2010) houve a realização da adaptação e tradução cultural da Breeastfeeding Self-Efficacy Scale no Brasil a partir da aplicação para a verificação das propriedades psicométricas do instrumento em uma população nordestina. Durante o processo de tradução e validação da escala para o contexto brasileiro os autores concluíram que a escala é capaz de permitir aos profissionais de saúde identificar o grau de eficácia das mães em amamentar, investigando possíveis vulnerabilidades e realizando tomada de conduta precoce para evitar o desmame precoce. Foi proposto ainda que houvesse a aplicação da BSES em outros contextos para consolidar a validação de suas propriedades psicométricas no Brasil.

Neste contexto Mariano et al., (2016) ao realizarem um estudo de aplicação da BSES em uma população de mulheres vítimas de violência pelo parceiro íntimo em Ribeirão Preto, detectaram escores de autoeficácia na amamentação de $54 \%$ alta, 34\% média e $12 \%$ baixa na população estudada, não havendo associação estatística do grau de autoeficácia e os tipos de violência sofrida pelas respectivas mulheres durante a gestação, mostrando a aplicabilidade da escala validada naquele contexto.

No estudo realizado por Abuchaim et al., (2016) com puérperas de um centro de incentivo e apoio ao aleitamento materno de São Paulo aplicando a Breastfeeding Self-Efficacy Scale (BSES) para a verificação da prevalência da autoeficácia em amamentar e a associação desta com a ocorrência de depressão pósparto, observou-se as taxas de autoeficácia foram $36,6 \%$ alta, $24,4 \%$ baixa e 39 , 9\% média, com predomínio elevado de escore no domínio técnico na autoeficácia em amamentar, com média entre as mulheres de 76,53 de pontuação mostrando que as puérperas possuem maior habilidade técnica, diferente do pensamento intrapessoal que revela um resultado de 52,74. Quando se realizou a associação entre a autoeficácia para amamentar e a ocorrência de depressão pós-parto (DPP), observou-se a autoeficácia moderada ou alta diminui em 27,4 ou 38,8 respectivamente os escores de DPP, por outro lado elevados índices nos escores de depressão diminuíram em 11,84 pontos o escore de auto eficácia na amamentação.

Em outro contexto Guimarães et al., (2017a) ao compararem a autoeficácia na amamentação entre puérperas adolescentes e adultas, em uma maternidade de Ribeirão Preto, aplicando a BSES, foi possível observar que o escore de pontuação média das puérperas adolescentes foi de 138,86, indicando auto eficácia alta e entre as adultas, a média de pontuação foi de 137,7, indicando auto eficácia moderada, mostrando um limite inferior bem próximo da auto eficácia alta. Por outro lado, não houve diferença estatística significativa entre as médias de pontuação global entre esses dois grupos, ou seja, os níveis da autoeficácia na amamentação foram semelhantes.

Já em outro estudo de aplicação da BSES realizado por Guimarães et al., (2017) para a verificação de fatores relacionados à auto eficácia na amamentação no pós-parto imediato entre puérperas adolescentes de Ribeirão Preto, evidenciou classificação de auto eficácia na amamentação de 11,70\%, 34,4\% e 54,26\% baixa, moderada e alta respectivamente. Além disso, o estudo mostrou que houve associação significativa entre a variável "auxilio nos cuidados com o bebe" e a "auto eficácia na amamentação"; ou seja, as adolescentes que referiram receber ajuda de suas mães e /ou sogras tiveram maior nível de auto eficácia na amamentação, apresentando um escore de $54,26 \%$ relativo a auto eficácia alta.

Nesta mesma linha de raciocínio Conde et al., (2017) utilizaram a BSES para analisar a duração do aleitamento materno exclusivo e a auto eficácia da amamentação entre mães adolescentes de uma maternidade pública de Ribeirão Preto revelou percentuais de auto eficácia de 56,90\% alto, $35 \%$ moderado e $8,10 \%$ baixo. Já a prevalência de adolescentes que mantiveram AME em até 30 dias após o parto $62 \%$; $52,59 \%$ mantiveram AME em até 60 dias após o parto e 16\% mantiveram o AME em até 180 dias. Por outro lado, observou-se que não houve diferença estatisticamente significativa entre a duração do aleitamento materno exclusivo nos períodos delimitados com os percentuais de auto eficácia.

\section{CONCLUSÃO}

A partir da realização da presente pesquisa, foi possível perceber que a BSES é um instrumento de relevância no que diz respeito à apreensão da autoeficácia de mães para amentar e por conseguinte para a verificação dos padrões de aleitamento materno exclusivo, uma vez que estes estão intimamente ligados à escores presentes na referida escala. $\mathrm{Na}$ análise dos estudos desta revisão, pode-se perceber que a versão 
brasileira da BSES é perfeitamente aplicável em diferentes contextos, sendo inclusive utilizada para comparações entre diferentes grupos populacionais e a autoeficácia em contextos diversos, como a depressão pós-parto e a situação de mulheres vítimas de violência pelo parceiro. Neste contexto a efetivação e a aplicação da BSES nos espaços assistenciais onde se verifica o cotidiano do binômio mãe-bebê pode proporcionar parâmetros que levarão o profissional avaliador a perceber fatores que possam interferir na autoeficácia do aleitamento materno e assim utilizar estratégias de promoção da amamentação.

\section{REFERÊNCIAS}

1. ABUCHAIM ESV, CALDEIRA NT, DI LUCCA MM et al. Depressão pós-parto e autoeficácia materna para amamentar: prevalência e associação. Acta Paulista de Enfermagem, 2016; 29 (6): 664-670.

2. BOCCOLINI CS, BOCCOLINI PMM, MONTEIRO FR et al. Tendência de indicadores do aleitamento materno no Brasil em três décadas. Revista de Saúde Pública, 2017; 51:108.

3. Brasil. Ministério da Saúde. Secretaria de Atenção à Saúde. Departamento de Atenção Básica. Caderno de atenção básica, № 23. 2 ed. rev - Brasília, 2009. 184 p.

4. CONDE RG, GUIMARÃES CMSG, GOMES-SPONHOLZ FA et al. Autoeficácia na amamentação e duração do aleitamento materno exclusivo entre mães adolescentes. Acta Paulista de Enfermagem, 2017; 30(4):3839.

5. ERCOLE FF, MELO LS, ALCOFORADO CLGC. Revisão integrativa versus Revisão Sistemática. Reme: Revista Mineira de Enfermagem, 2014; 18(1): 1-260.

6. GUIMARÃES CMS, CONDE RG, BRITO BC et al. Comparação da autoeficácia na amamentação entre puérperas adolescentes e adultas em uma maternidade de Ribeirão Preto, Brasil. Texto \& Contexto Enfermagem, 2017; 26(1): e4100015.

7. GUIMARÃES CMS, CONDE RG, GOMES-SPONHOLZ FA et al. Fatores relacionados à autoeficácia na amamentação no pós-parto imediato entre puérperas adolescentes. Acta Paulista de Enfermagem, 2017; 30(1): 109-15.

8. MARIANO LMB, MONTEIRO JCS, SEFANELLO J et al. Aleitamento materno exclusivo e autoeficácia materna entre mulheres em situação de violência por parceiro intímo. Texto \& Contexto - Enfermagem, 2016; 25(4): e2910015.

9. MORAES BA, GONÇALVES AC, STRADA RKJ et al. Fatores associados à interrupção do aleitamento materno exclusivo em lactentes com até 30 dias. Revista Gaúcha de Enfermagem,2016; 37 (esp): e0044.

10. NUNES LM. Importância do aleitamento materno na atualidade. Boletim Científico de Pediatria. 2015;04(3):55-8.

11. ORIA MOB, XIMENES LB. Tradução e adaptação cultural da Breastfeeding Self-Efficacy Scale para o português. Acta Paulista de Enfermagem, 2010;23(2):230-238.

12. VICTORA CG, BARROS AJD, FRANÇA GVA et al. Amamentação no século XXI: epidemiologia, mecanismos, e efeitos ao longo da vida. Revista Epidemiologia e Serviço de Saúde. Brasília. 2016. 